\title{
Sintomas depressivos, depressão e atividade física no envelhecimento: uma revisão integrativa
}

\author{
Depressive symptoms, depression and physical activity in elderly individuals: an integrating review
}

Síntomas depresivos, depresión y actividad física en el envejecimiento: una revisión integrativa

\section{RESUMO}

Objetivo: 0 presente estudo teve como objetivo realizar uma revisão integrativa da literatura sobre a prática de atividade física e os sintomas depressivos/depressão em idosos. Utilizou-se revisões e artigos completos publicados nas bases de dados PubMed e LILACS. Método: A presente revisão seguiu seis etapas: identificação do tema e elaboração da questão norteadora da pesquisa, estabelecimento dos critérios de inclusão e exclusão, extração de dados e categorização dos estudos, análise e síntese dos resultados e apresentação da revisão. A busca dos artigos foi realizada no período de 2007 a 2017 e foram identificados 253 estudos, porém utilizaram-se como amostra na presente revisão, 11 estudos. Resultados: Os resultados mostraram que a atividade física tem um efeito positivo na prevenção e redução dos sintomas depressivos/depressão, havendo uma relação inversa, entre estas duas variáveis, na maioria dos estudos. Conclusão: Portanto, a atividade física é uma medida preventiva importante para a melhoria da saúde mental dos idosos.

DESCRITORES: Sintomas depressivos. Idosos. Atividade física.

\section{ABSTRACT}

Objective: The present study aimed to carry out an integrative review of the literature on the practice of physical activity and depressive symptoms / depression in the elderly. Full articles and reviews published in the PubMed and LILACS databases were used. Method: The present review followed six steps: identification of the theme and elaboration of the guiding question of the research, establishment of the inclusion and exclusion criteria, data extraction and categorization of the studies, analysis and synthesis of the results and presentation of the review. The search for articles was carried out from 2007 to 2017 and 253 studies were identified, however 11 studies were used as a sample in this review. Results: The results showed that physical activity has a positive effect on the prevention and reduction of depressive symptoms / depression, with an inverse relationship between these two variables in most studies. Conclusion: Therefore, physical activity is an important preventive measure for improving the mental health of the elderly.

DESCRIPTORS: Depressive symptoms. Elderly. Physical activity.

\section{RESUMEN}

Objetivo: El presente estudio tuvo como objetivo realizar una revisión integradora de la literatura sobre la práctica de actividad física y síntomas depresivos / depresión en ancianos. Se utilizaron artículos completos y revisiones publicados en las bases de datos PubMed y LILACS. Método: La presente revisión siguió seis pasos: identificación del tema y elaboración de la pregunta orientadora de la investigación, establecimiento de los criterios de inclusión y exclusión, extracción de datos y categorización de los estudios, análisis y síntesis de los resultados y presentación de la revisión. . La búsqueda de artículos se realizó de 2007 a 2017 y se identificaron 253 estudios, sin embargo se utilizaron 11 estudios como muestra en esta revisión. Resultados: Los resultados mostraron que la actividad física tiene un efecto positivo en la prevención y reducción de los síntomas depresivos / depresión, con una relación inversa entre estas dos variables en la mayoría de los estudios. Conclusión: Por tanto, la actividad física es una importante medida preventiva para mejorar la salud mental de las personas mayores.

DESCRIPTORES: Síntomas depresivos. Anciano. Actividad física.

RECEBIDO EM: 28/02/2021 APROVADO EM: 22/03/2021

\section{Mara Jordana Magalhães Costa}

Doutora em Saúde Pública - FSP/USP. Licenciada em Educação Física - UESPI. Professora Adjunta do curso de Educação Física - DEF/UFPI.

ORCID: 0000-0003-2185-3814. 


\section{Ana Teresa de Abreu Ramos-Cerqueira}

Mestrado e Doutorado em Psicologia (Psicologia Experimental) pela Universidade de São Paulo. Psicóloga pela Pontifícia Universidade Católica de Campinas (PUCCAMP). Professora Associada aposentada e professora voluntária, da Universidade Estadual Paulista Júlio de Mesquita Filho (UNESP), junto ao Departamento de Neurologia, Psicologia e Psiquiatria da Faculdade de Medicina de Botucatu.

ORCID: 0000-0003-2374-8890

\section{INTRODUÇÃO}

A transição demográfica ocorrida no país vem aumentando a preocupação com a população idosa. Vasconcelos e Gomes ${ }^{1}$ ressaltaram que o país ainda se encontra em transição e em rápido processo de envelhecimento, apresentando novos desafios com vistas a se ampliar e aprimorar a atenção à saúde dos idosos.

Fabbri et. $\mathrm{al}^{2}$ ressaltam que no envelhecimento ocorre o progressivo acúmulo de múltiplas doenças, e ocorre de forma ainda mais acelerada em idades mais avançadas. Apontam ainda que os mesmos mecanismos que geram o envelhecimento também geram doenças crônicas relacionadas com a idade.

A depressão está entre as doenças mais prevalentes entre os idosos, sendo um importante problema de saúde mental tanto pela sua frequência, como pela sua forte associação com outras doenças e incapacidades que trazem prejuízos na qualidade de vida e sobrecarga para a família e para os serviços de saúde 3 . É considerada um transtorno de humor ou da área afetiva que pode afetar as pessoas em qualquer faixa etária, podendo ocasionar limitação funcional, tendo no idoso, formas de apresentação e etiologia heterogêneas porque envolvem aspectos biológicos relacionados com a fragilidade, comorbidade, aspectos psicológicos relacionados com a viuvez, mudança de papéis e aspectos sociais relacionados com a solidãó ${ }^{4}$.

Segundo Schimdt e Duncan et $\mathrm{l}^{5}$ a maior parte da carga originada de transtornos neuropsiquiátricos se deve à depressão, às psicoses e aos transtornos atribuíveis ao uso abusivo do álcool. Os transtornos neuropsiquiátricos e musculoesqueléticos são responsáveis por aproximadamente $25,0 \%$ da carga das Doenças Crônicas Não Transmissíveis (DCNT) no Brasil e isso requer uma atenção espe-
A transição

demográfica

ocorrida no país

vem aumentando a

preocupação com

a população idosa.

Vasconcelos e

Gomes ressaltaram

que o país ainda

se encontra em

transição e em

rápido processo de

envelhecimento,

apresentando

novos desafios com

vistas a se ampliar e

aprimorar a atenção à

saúde dos idosos. cial, uma vez que o mesmo indivíduo pode apresentar múltiplas comorbidades.

A prevalência de depressão nos idosos pode diferir substancialmente dependendo da definição utilizada para sua operacionalização, do instrumento usado para sua detecção e, principalmente, da população estudada ${ }^{6}$.

Estudo realizado em sete países de baixa e média renda por Albanese et $\mathrm{al}^{7}$ sobre depressão em idosos, mostrou que a prevalência de depressão variou de $0,5 \%$ na China para 13,8\% em República Dominicana, de acordo com os critérios diagnósticos da CID-10. Utilizando critérios de outra classificação, EURO-D encontrou-se prevalência ainda mais elevada, que variou de 2,8\% na China para $41,5 \%$ na Índia. Cong et $\mathrm{al}^{8}$ mostraram em pesquisa realizada com objetivo de verificar a prevalência e fatores de risco de depressão para idosos na cidade de Fuzhou (China), valores de $10,5 \%$ para depressão, $9,4 \%$ para levemente deprimido, e $1,1 \%(\mathrm{n}=21)$ para gravemente deprimido.

Uma alternativa importante para manutenção da qualidade de vida do indivíduo idoso é a prática de atividade física regular, sendo esta caracterizada por um movimento corporal realizado pela musculatura esquelética e que resulte em um gasto energético acima dos níveis de repouso, podendo ser avaliada e domínios como o ocupacional, lazer, atividades domésticas e transportes ${ }^{9}$. Esta prática tem se mostrado como protetora contra diversos problemas que acometem o idoso, dentre eles, o comprometimento funcional, desempenho cognitivo e depressão ${ }^{10}$.

Assim, observa-se que a atividade física pode ser considerada como um fator importante para modificar o comportamento do idoso, melhorando não apenas sua autonomia funcional, mas também podendo reduzir os sintomas depressivos. 


\section{artigo}

Frente ao exposto, faz-se a seguinte questão de pesquisa: Quais as evidências científicas sobre a associação entre a prática de exercício físico e sintomas depressivos/depressão em indivíduos idosos? Para responder a esta indagação, a presente pesquisa teve como objetivo realizar uma revisão integrativa da literatura sobre a prática de atividade física e os sintomas depressivos/depressão em idosos.

\section{MÉTODOS}

Utilizou-se a revisão integrativa de produções científicas na área do envelhecimento, que estudaram a associação entre sintomas depressivos/depressão e atividade física.

A presente revisão seguiu seis etapas: identificação do tema e elaboração da questão norteadora da pesquisa, estabelecimento dos critérios de inclusão e exclusão, extração de dados e categorização dos estudos, análise e síntese dos resultados e apresentação da revisão ${ }^{11}$.

A busca dos artigos foi realizada no período de 2007 a 2017. Os critérios de inclusão estabelecidos para este estudo foram: revisões e artigos completos publicados nos idiomas português, inglês e espanhol nas bases de dados PubMed e LILACS e que utilizassem como instru-
Figura 1: Fluxograma do processo de seleção dos estudos - São Paulo, SP, Brasil, 2019.

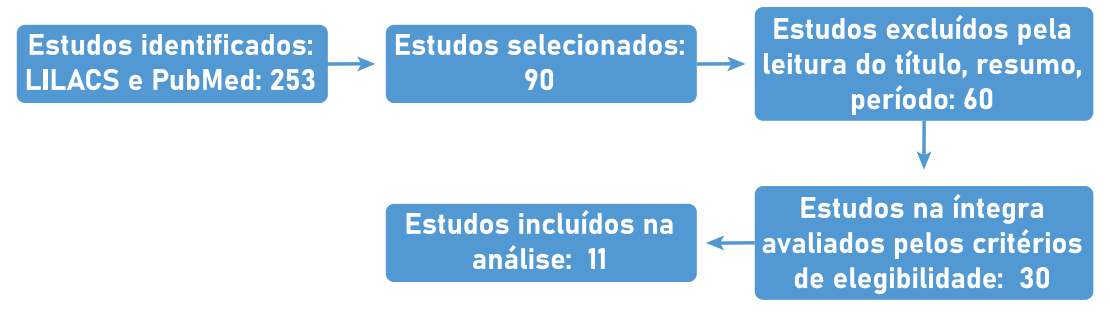

mento a Escala de Depressão Geriátrica (GDS). Os critérios de exclusão estabelecidos foram: relatos de experiências, resenhas, editoriais, dissertações, teses e monografias; resumos publicados em anais de eventos; artigos que não possuíssem relação direta com o tema; artigos repetidos nas bases de dados.

Os descritores utilizados na busca foram selecionados de acordo com a temática do estudo, por meio dos Descritores em Ciências da Saúde (DeCS) e do Medical Subject Heading (MESH). Os descritores foram utilizados em inglês e em português. Para a estratégia de busca foi utilizado o operador booleano "OR", com os descritores: aged/aging "OR" depressive disorder "OR" motor activity, sintomas depressivos, atividade física e idosos. Os artigos foram escolhidos pela leitura do título e resumos presentes nas bases de dados que fossem pertinentes à questão de pesquisa.

O fluxograma (figura 1) descreve o percurso de identificação, seleção e inclusão dos estudos selecionados, segundo a base de dados consultada.

Quadro 1. Síntese dos estudos selecionados segundo o título, ano, base de dados, objetivo, local do estudo, instrumentos utilizados, delineamento e principais resultados (2007-2017). São Paulo, SP, Brasil, 2019.

\begin{tabular}{|c|c|c|c|}
\hline ESTUDO & TÍTULO & OBJETIVO & PRINCIPAIS RESULTADOS \\
\hline E1 & $\begin{array}{l}\text { The Impact of Resistan- } \\
\text { ce Exercise Training on } \\
\text { the Mental Health of Ol- } \\
\text { der Puerto Rican Adults } \\
\text { With Type } 2 \text { Diabetes }\end{array}$ & $\begin{array}{c}\text { Determinar o impacto de um programa de } \\
\text { treinamento de exercícios de resistência } \\
\text { progressiva (PRT) de } 16 \text { semanas de alta } \\
\text { intensidade sobre a saúde mental de adultos } \\
\text { mais velhos com diabetes tipo } 2 \text {. }\end{array}$ & $\begin{array}{l}\text { Após a intervenção de } 16 \text { semanas com exercício } \\
\text { de resistência progressiva, houve fortes diferenças } \\
\text { estatisticamente significativas entre o grupo que } \\
\text { realizava exercício de resistência e o grupo contro- } \\
\text { le, mostrando melhorias em ambas as médias da } \\
\text { GDS (3,1 } \pm 3,5 \text { vs. } 12,4 \pm 8) \text {. }\end{array}$ \\
\hline E2 & $\begin{array}{l}\text { Physical activity and } \\
\text { depressive symptoms } \\
\text { in community-dwelling } \\
\text { elders from southern } \\
\text { Brazil }\end{array}$ & $\begin{array}{l}\text { Determinar a relação entre atividade } \\
\text { e sintomas depressivos em idosos } \\
\text { residentes na comunidade }\end{array}$ & $\begin{array}{l}\text { Foi observada tendência a menor prevalência de } \\
\text { sintomas depressivos em indivíduos com níveis } \\
\text { mais altos de atividade física entre os homens, } \\
\text { mas não entre as mulheres ( } p=0,04,0,03 \text { e 0,36, } \\
\text { respectivamente). }\end{array}$ \\
\hline E3 & $\begin{array}{l}\text { Fatores associados aos } \\
\text { sintomas depressivos } \\
\text { em idosos: estudo Epi } \\
\text { Floripa }\end{array}$ & $\begin{array}{l}\text { Analisar a prevalência e fatores associados a } \\
\text { sintomas depressivos em idosos. }\end{array}$ & $\begin{array}{c}\text { Menor prevalência de sintomas depressivos foi } \\
\text { observada em idosos que relataram: ter relação } \\
\text { sexual (RP=0,43), trocar mensagens pela internet } \\
(\mathrm{RP}=0,34) \text {, consumo alto ou moderado de álcool } \\
(\mathrm{RP}=0,60 ; 0,59 \text { respectivamente), participar de } \\
\text { grupos de convivência ou religiosos ( } \mathrm{RP}=0,71) \text {, se- } \\
\text { rem ativos no lazer ( } \mathrm{RP}=0,48) \text { e ter relacionamento } \\
\text { mensal com amigos ou parentes ( } \mathrm{RP}=0,56 ; 0,69 \text {, } \\
\text { respectivamente). }\end{array}$ \\
\hline
\end{tabular}




\begin{tabular}{|c|c|c|c|}
\hline E4 & $\begin{array}{l}\text { Características socio- } \\
\text { demográficas e hábitos } \\
\text { de vida de idosos com } \\
\text { e sem indicativo de } \\
\text { depressão }\end{array}$ & $\begin{array}{c}\text { Comparar as variáveis sociodemográficas e } \\
\text { hábitos de vida dos idosos com e sem indica- } \\
\text { tivo de depressão }\end{array}$ & $\begin{array}{l}\text { O percentual de idosos que não praticavam ativi- } \\
\text { dade física foi, proporcionalmente, maior entre os } \\
\text { que apresentavam indicativo de depressão quando } \\
\text { comparados àqueles sem indicativo ( } 2=8,369 \text {; } \\
\text { p=0,004), mostrando a importância da prática de } \\
\text { atividades físicas para a saúde mental. }\end{array}$ \\
\hline E5 & $\begin{array}{l}\text { Avaliação dos níveis de } \\
\text { depressão em idosos } \\
\text { praticantes de diferen- } \\
\text { tes exercícios físicos }\end{array}$ & $\begin{array}{c}\text { Avaliar o nível de depressão em idosos } \\
\text { pratican-tes de diferentes tipos de exercícios } \\
\text { físicos. }\end{array}$ & 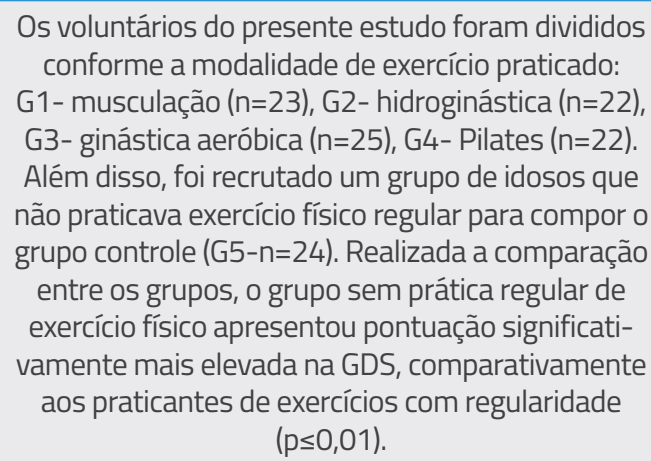 \\
\hline E6 & $\begin{array}{l}\text { Qualidade de vida e } \\
\text { sintomas depressivos } \\
\text { em idosos de três faixas } \\
\text { etárias praticantes de } \\
\text { atividade física }\end{array}$ & $\begin{array}{l}\text { Verificar se existe diferença entre sintomas } \\
\text { depressivos e qualidade de vida em idosos } \\
\text { de diferentes faixas etárias e praticantes de } \\
\text { atividade física, por meio dos protocolos: } \\
\text { Geriatric Depression Scale (GDS-15) e SF-36. }\end{array}$ & $\begin{array}{l}\text { Os resultados apontaram que os idosos ativos } \\
\text { apresentaram diferenças entre as médias nas } \\
\text { variáveis sintomas depressivos e qualidade de } \\
\text { vida, mas sem diferença significativa entre as três } \\
\text { faixas etárias. }\end{array}$ \\
\hline E7 & $\begin{array}{l}\text { Physical Activity and } \\
\text { Depressive Symptoms } \\
\text { Interact to Predict } \\
\text { Executive Functioning } \\
\text { Among Community } \\
\text { Dwelling Older Adults }\end{array}$ & $\begin{array}{l}\text { Testar os efeitos da atividade física, sinto- } \\
\text { mas depressivos e funcionamento executivo } \\
\text { com dados longitudinais de múltiplas ondas } \\
\text { de uma amostra de idosos da comunidade } \\
\text { com níveis geralmente baixos de sintomas } \\
\text { depressivos. }\end{array}$ & $\begin{array}{c}\text { Aqueles que relataram maior atividade física do } \\
\text { que outros tiveram funcionamento executivo (inclui } \\
\text { memória de trabalho, inibição e troca de tarefas) } \\
\text { melhor ( }=0,42, \mathrm{SE}=0,19, \mathrm{t}(102)=2,14, \mathrm{p}<0,05 \text { ). } \\
\text { Mostrou ainda que atividade física foi mais pro- } \\
\text { tetora do funcionamento executivo no avaliados, } \\
\text { durante períodos de sintomas depressivos relati- } \\
\text { vamente elevados. }\end{array}$ \\
\hline E8 & $\begin{array}{l}\text { Participation in Physical, } \\
\text { Social, and Religious } \\
\text { Activity and Risk of De- } \\
\text { pression in the Elderly: } \\
\text { A Community-Based } \\
\text { Three-Year Longitudinal } \\
\text { Study in Korea }\end{array}$ & $\begin{array}{l}\text { Avaliar a associação longitudinal entre } \\
\text { participação em atividades físicas, sociais e } \\
\text { religiosas e o risco de depressão em idosos. }\end{array}$ & $\begin{array}{l}\text { Análise de regressão logística indicou que idosos } \\
\text { que participaram de atividade física tiveram } \\
\text { uma OR ajustada de } 0,81 \text {, em comparação com } \\
\text { participantes que não realizavam esta atividade, } \\
\text { mostrando que uma participação na atividade } \\
\text { física, foi associada com diminuição do risco de } \\
\text { depressão nos idosos, após ajuste por fatores de } \\
\text { confusão conhecidos. }\end{array}$ \\
\hline E9 & $\begin{array}{l}\text { Physical benefits and } \\
\text { reduction of depressive } \\
\text { symptoms among the } \\
\text { elderly: Results from } \\
\text { the Portuguese "Natio- } \\
\text { nal Walking Program" }\end{array}$ & $\begin{array}{l}\text { verificar as alterações nos idosos após a } \\
\text { participação no Programa Nacional de Marcha } \\
\text { e Corrida de Portugal, em relação ao nível de } \\
\text { aptidão física, medidas antropométricas, bem } \\
\text { como os sintomas depressivos }\end{array}$ & $\begin{array}{l}\text { A intervenção com a prática de atividade física teve } \\
\text { a efeito na remissão de sintomas depressivos ( } \mathrm{x} \text {. } \\
\qquad 0,001) .\end{array}$ \\
\hline E10 & $\begin{array}{l}\text { Prevalência de sinto- } \\
\text { mas depressivos em } \\
\text { idosas de um Centro de } \\
\text { Referência de Atenção } \\
\text { ao Idoso no município } \\
\text { de Passo Fundo, Rio } \\
\text { Grande do Sul }\end{array}$ & $\begin{array}{l}\text { Analisar a prevalência de sintomas depres- } \\
\text { sivos em idosas e fatores associados a essa } \\
\text { patologia, em um Centro de Referência e } \\
\text { Atenção ao Idoso do município de Passo } \\
\text { Fundo, Rio Grande do Sul. }\end{array}$ & $\begin{array}{c}\text { Dos } 313 \text { idosos avaliados, foi observado que quan- } \\
\text { to aos níveis de atividade física suficientemente } \\
\text { ativo e muito ativo, foram encontrados em } 181 \\
(57,9 \%) \text { das idosas. Na análise bivariada, a maior } \\
\text { prevalência de depressão esteve associada às } \\
\text { idosas classificadas como insuficientemente ativa } \\
(10,6 \% ; p=0,033) .\end{array}$ \\
\hline
\end{tabular}




\begin{tabular}{|c|c|c|c|}
\hline E11 & $\begin{array}{c}\text { Depressive symptoms } \\
\text { and associated factors } \\
\text { in elderly people in the } \\
\text { Primary Health Care }\end{array}$ & $\begin{array}{c}\text { Analisar os sintomas depressivos e seus } \\
\text { fatores associados, ao uso de antidepressivos } \\
\text { em idosos com diagnóstico de depressão. }\end{array}$ & $\begin{array}{c}\text { Os resultados mostraram que dos 248 idosos } \\
\text { pesquisados, 59,3\% não pratica atividade física e } \\
\text { destes } 42,2 \% \text { apresentaram maiores chances de } \\
\text { ter a depressão (42,3\%) quando comparado com os } \\
\text { que praticam atividade física }(18,4 \%) \text { p<0.001. }\end{array}$ \\
\hline Fonte: Dados da Pesquisa, 2019.
\end{tabular}

\section{RESULTADOS}

Foram selecionados 11 estudos que contemplaram o proposto pelo artigo e que se utilizaram da GDS para avaliar os sintomas depressivos. A síntese dos estudos selecionados e incluídos na revisão integrativa, estão abordados no Quadro 1.

\section{DISCUSSÕES}

Os resultados da presente revisão integrativa mostraram que a atividade física teve efeito positivo nos sintomas depressivos/depressão, havendo uma relação inversa entre estas duas variáveis.

Estudo de Borges, Benedetti e Mazo ${ }^{12}$ mostrou influência positiva do exercício físico sobre os sintomas depressivos e aptidão funcional, assim como também observou uma tendência na redução dos sintomas depressivos e no aumento da aptidão funcional. No estudo de Cheick et $\mathrm{al}^{13}$, os resultados mostraram que as atividades físicas realizadas no lazer de forma regular e orientada, apresentaram tendência de redução dos escores indicativos para a ansiedade e depressão em idosos.

Gumarães e Caldas ${ }^{14}$ realizaram um estudo de revisão sistemática no qual quinze artigos foram avaliados, a maioria mostrando os efeitos benéficos da atividade física nos sintomas depressivos/depressão, resultados que são similares aos da presente revisão.

Sabe-se que o sedentarismo é um grande problema de saúde pública e em muitos estudos mostra-se associado à incapacidade funcional, déficit cognitivo, e aos sintomas depressivos/depressão. Pode-se afirmar que a atividade física é importante para os idosos, melhorando seu estado de humor, sugerindo assim que o desempenho de atividade física,
Nesta perspectiva,

Stella et al.

ressalta que a

atividade física,

quando regular

e bem planejada,

contribui para a

minimização do

sofrimento psíquico

do idoso deprimido,

além de oferecer

oportunidade

de envolvimento

psicossocial, elevação

da autoestima,

implementação das

funçôes cognitivas,

com saída do quadro

depressivo e menores

taxas de recaída. necessariamente, deveria fazer parte da abordagem terapêutica preventiva e curativa da depressão. A reversibilidade da depressão em idosos associada ao sedentarismo está em discussão e mais estudos são necessários para fornecer indicações de uma abordagem profilática para estes pacientes ${ }^{15}$.

Nesta perspectiva, Stella et $\mathrm{al}^{16}$ ressalta que a atividade física, quando regular e bem planejada, contribui para a minimização do sofrimento psíquico do idoso deprimido, além de oferecer oportunidade de envolvimento psicossocial, elevação da autoestima, implementação das funções cognitivas, com saída do quadro depressivo e menores taxas de recaída.

O estudo E1 e E9 foram pesquisas de intervenção e todas elas mostraram uma redução dos escores de depressão/sintomas depressivos, após a intervenção com a prática de atividade física. Em estudo de revisão sistemática de Villada, Vélez e Baena ${ }^{17}$, todos os estudos selecionados (11) foram experimentais mostrando que a maioria dos estudos incluídos na revisão sistemática indicaram efeito benéfico de diferentes modalidades de atividade física e exercício nos sintomas depressivos de adultos mais velhos (acima de 60 anos) diagnosticados com depressão. Os autores ainda concluem que o exercício aeróbico pode beneficiar idosos com depressão, sem clareza sobre o tempo necessário para atingir o efeito ou a dose deve receber estes pacientes. As atividades físicas mistas, que combinam trabalho aeróbico, força, alongamento, equilíbrio, e outras modalidades de exercícios parece reduzir o nível de depressão e devem ser recomendadas na abordagem terapêutica dessas pessoas, embora ainda não saiba a melhor combinação, dose ou duração da intervenção, o que necessita de mais investigações. 
Estudo transversal realizado em idosos de Karachi no Pakistan, também reforça o efeito positivo da prática de atividades físicas e a prevenção da depressão em idosos ${ }^{18}$. Observou-se que idosos que realizavam atividades físicas acima de 310 minutos por semana tiveram $60 \%$ menos chance de ter depressão quando comparados com aqueles que realizavam menos do que 120 minutos semanais (OR Ajustada $=0.4 ; \mathrm{IC} 95 \%=0.2-0.7)$. Os autores reforçam que a atividade física é um método de baixo custo, tanto na promoção preventiva da saúde, bem como estratégia de auto gestão para abordar a saúde mental dos idosos, especialmente em idosos de baixa e média renda em países como o Paquistão ${ }^{18}$.
Os estudos que tratam da relação entre sintomas depressivos/depressão e a prática da atividade física em idosos não compóe um grande acervo na literatura, sendo, portanto, um assunto relevante e que necessita de maiores investigações. O que se pôde observar dentre a maioria dos estudos analisados na presente revisão, é que a intervenção com a prática de atividade física demonstrou uma medida não farmacológica para idosos que tem sintomas depressivos/depressão. E esta atividade pode ser aeróbica, de força ou de flexibilidade. Os estudos não procuram ainda mostrar qual a melhor atividade, mas que a realização das atividades físicas, especialmente as realizadas em grupos, é importante para o tratamento e remissão dos sintomas depressivos/depressão em idosos.

\section{CONCLUSÃO}

Pode-se concluir que os estudos revisados neste trabalho mostraram os efeitos benéficos da atividade física e do exercício para prevenção e redução de sintomas depressivos em pessoas idosas, contribuindo para aprofundar o conhecimento científico na temática abordada e fortalecendo o efeito da atividade física enquanto tratamento não farmacológico em idosos com sintomas depressivos.

Assim, sugerem-se mais pesquisas sobre o assunto que apresentem um maior rigor metodológico na abordagem do processo, particularmente da análise de dados, resultando assim, na diminuição de vieses e erros.

\section{REFERÊNCIAS}

1. Vasconcelos AMN, Gomes MMF. Transição demográfica: a experiência brasileira. Epidemiol Serv Saúde 2012; 21(4):539-548.

2. Fabbri E, Zoli M, Gonzalez-Freire M, Salive ME, Studenski SA, Ferrucci L. Aging and Multimorbidity: New Tasks, Priorities, and Frontiers for Integrated Gerontological and Clinical Research. J Am Med Dir Assoc 2015; 16(8):640-7.

3. Mendes-Chillof CL, Ramos-Cerqueira ATA. Estudo SABE - Sintomas depressivos em idosos do município de São Paulo. 2011 [Dissertação]. Botucatu; 2011.

4. Silva AR. Doenças crônicas não transmissíveis e sinais e sintomas de depressão e de declínio cognitivo em idosos na atenção primária à saúde. 2015. [Dissertação].Porto Alegre; 2015.

5. Albanese E. et al. No association between fish intake and depression in over 15,000 older adults from seven low and middle income countries--the 10/66 study. PloS one 2012;7(6): e38879.

6. Cong L. et al. Depression and Associated Factors in the Elderly Cadres in Fuzhou, China: A Community-based Study. International Journal of Gerontology 2015; 9(1): 29-33.

7. Hellwig N; Munhoz TN; Tomasi E. Sintomas depressivos em idosos: estudo transversal de base populacional. Ciência \& Saúde Coletiva 2016; 21(11): 3575-3584.

8. Schmidt Ml; Duncan BB. O enfrentamento das doenças crônicas não transmissíveis: um desafio para a sociedade brasileira. Epidemiologia e Serviços de Saúde 2011; 20(4): 421-423.

9. Tribess S, Virtuoso Júnior JS, Oliveira RF de. Atividade física como preditor da ausência de fragilidade em idosos. Rev Assoc Med Bras 2012; 58(3):341-347.

10. Landi F, Abbatecola AM, Provinciali M, Corsonello A, Bustac- chini S; Manigrasso L, Cherubini A, Bernabei R, Lattanzio F. Moving against frailty: does physical activity matter? Biogerontology 2010; 11:537-45.

11. Mendes KDS, Silveira RCCP, Galvão CM. Revisão integrativa: método de pesquisa para a incorporação de evidências na saúde e na enfermagem. Texto Contexto Enferm 2008;17(4):758-64.

12. Borges LJ, Benedetti TRB, Mazo GZ. Influencia del ejercicio físico en los síntomas depresivos y en la aptitud funcional de ancianos en el sur de Brasil. Rev Esp Geriatr Gerontol 2010;45(2):72 $-78$.

13. Cheik NC, Reis IT, Heredia RAG, Ventura ML, Tufik S, Antunes HKM, Mello MT. Efeitos do exercício físico e da atividade física na depressão e ansiedade em indivíduos idosos. R bras Ci e Mov 2003; 11(3): 45-52.

14. Gumarães JM, Caldas CP. A influência da atividade física nos quadros depressivos de pessoas idosas: uma revisão sistemática. Rev Bras Epidemiol 2006; 9(4): 481-92.

15. Ciucurel C, Iconarua EL. The importance of sedentarism in the development of depression in elderly people. Procedia - Social and Behavioral Sciences 2012; 33: 722 - 726.

16. Stella F, Gobbi S, Corazza DI, Costa JLR. Depressão no Idoso: Diagnóstico, Tratamento e Benefícios da Atividade Física. Motriz 2002; 8(3): 91-98.

17. Villada FAP, Vélez EFA, Baena LZ. Ejercicio físico y depresión en adultos mayores: una revisión sistemática. Rev Colomb Psiquiat 2013;42(2):198-211.0

18. Bhamani, MA. Khan, MM. Karim, MS. Depression and its association with functional status and physical activity in the elderly in Karachi, Pakistan. Asian Journal of Psychiatry 2015;14: 46-51. 